Ciencia y Educación, Vol. 5, No. 3, septiembre-diciembre, 2021

ISSN (impreso): 2613-8794・ISSN (en línea): 2613-8808

DOI: https://doi.org/10.22206/cyed.2021.v5i3.pp61-78

\title{
Alfabetización inicial en tiempos de covid-19. Retos de la docencia a distancia
}

\author{
Early literacy in times of covid-19. Challenges \\ of distance teaching
}

Evangelina Cervantes Holguín, ${ }^{a}$ ORCID: 0000-0001-6980-2210

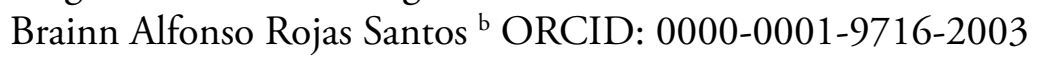

Recibido: 24/04/21 • Aprobado: 28/06/2021

Cómo citar: Cervantes Holguín, E., \& Rojas Santos, B. A. (2021). Alfabetización inicial en tiempos de covid-19. Retos de la docencia a distancia. Ciencia y Educación, 5(3), 61-78. Doi: https://doi.org/10.22206/cyed.2021.v5i3.pp61-78

\section{Resumen}

El presente estudio tiene como objetivo recuperar la experiencia del profesorado de primer ciclo de educación primaria en torno a los saberes, las prácticas y las adecuaciones implicadas en los procesos de alfabetización inicial en tiempos de emergencia sanitaria. A partir de una investigación cualitativa de tipo estudio de caso, se entrevistó a 13 docentes radicados en el estado de Chihuahua, México. En la experiencia concreta, el profesorado puso en juego una serie de saberes docentes que, puestos a prueba en la pandemia, se fortalecieron a partir de su propia iniciativa, el trabajo colectivo entre pares y la solidaridad de las familias para afrontar los problemas, superar las carencias y actuar en favor de los estudiantes. En el balance, los docentes reconocen debilidades en la enseñanza de la lectura y la escritura a distancia, por lo que se requieren investigaciones que orienten el diseño de acciones para fortalecer las competencias docentes.

Palabras clave: alfabetización, educación básica, justicia social, pandemia, práctica pedagógica.

\begin{abstract}
The objective of this study is to recover the experience of teachers in the first cycle of primary education regarding the knowledge, practices and adjustments involved in the processes of early literacy in health emergency times. From a qualitative research case study type, 13 teachers based in the state of Chihuahua, Mexico were interviewed. In concrete experience, the teachers put into play a series of teaching knowledge that, put to the test in the pandemic, was strengthened from their own initiative, collective work among peers and the solidarity of families to face problems, overcome deficiencies and act in favor of the students. In the balance, teachers recognize weaknesses in teaching literacy and remote work, so research to guide the design of actions to strengthen teaching skills are required.
\end{abstract}

Keywords: Literacy, basic education, social justice, pandemics, teaching practice.

\footnotetext{
a Universidad Autónoma de Ciudad Juárez, México, Correo-e: evangelina.cervantes@uacj.mx,

b Instituto Tecnológico de Estudios Superiores de Monterrey, Ciudad Juárez, México. Correo-e: a01687035@itesm.mx,
} 


\section{Introducción}

A nivel internacional, la alfabetización constituye un elemento clave para la movilidad social, la dignidad y el ejercicio de los derechos humanos (Organización de las Naciones Unidas (ONU), 2020). En el mundo actual, leer y escribir amplía las posibilidades de progreso, participación y agencia de las personas. Para Martin (2020), la alfabetización ofrece múltiples beneficios humanos, culturales, sociales y económicos que, en conjunto, nutren la vida individual y colectiva. Pese a su importancia, hoy 773 millones de adultos en el mundo carecen de las competencias básicas en lectoescritura (Organización de las Naciones Unidas para la Educación, la Ciencia y la Cultura (UNESCO), 2020).

En México, según el último informe del Programa para la Evaluación Internacional de Alumnos (PISA, por sus siglas en inglés), el desempeño de los estudiantes se encuentra por debajo del promedio de la Organización para la Cooperación y el Desarrollo Económico (OCDE, 2019). Desde el gobierno federal, se reconoce el "papel central de la lectura y escritura en la construcción del pensamiento crítico y de nuevas formas de pensar y repensar el mundo más allá de lo inmediato" (Gobierno de México, 2020, p. 206); en correspondencia, el modelo educativo vigente asume entre sus principales metas generar las condiciones para que el alumnado de educación obligatoria consolide "conforme a su ritmo de aprendizaje, su dominio de la lectura, la escritura y las matemáticas" (Secretaría de Educación Pública (SEP), 2017a, p. 104).

En marzo de 2020, covid-19 convulsionó la vida social, en especial, los procesos educativos marcados históricamente por la desigualdad (Cervantes \& Gutiérrez, 2020). En el mundo los procesos educativos se interrumpieron: docentes y estudiantes se vieron obligados a transitar de espacios presenciales a entornos virtuales de aprendizaje a fin de continuar con las actividades escolares. En este rubro, los principales desafíos se vinculan con el rezago en el aprendizaje y el abandono escolar que han convocado la implementación de diversas acciones entre las que se destacan el ajuste al currículo, su flexibilización, la definición de contenidos prioritarios, la formación docente y diversas estrategias de apoyo (Comisión Nacional para la Mejora Continua de la Educación (MEJOREDU), 2020a). En la experiencia mexicana, luego de la declaración de emergencia sanitaria, el gobierno federal estableció diversas acciones - el programa Aprende en casa, basado en el uso masivo de la televisión, los materiales impresos y los programas a través de plataformas tecnológicas - para la continuidad de las tareas educativas que derivó en un cambio radical caracterizado por diversas:

carencias propias del sistema educativo, de sus actores principales y de las escuelas; unas tienen que ver con la disponibilidad de recursos, las condiciones de la infraestructura física, los equipamientos tecnológicos básicos y materiales educativos, las capacidades cognitivas y emocionales de los actores; otras, que son de orden institucional, se relacionan con la manera de enfrentar con mayor solvencia las demandas exigidas en el escenario de emergencia. (MEJOREDU, 2020a, p. 6)

A nivel nacional, la continuidad académica convocó al personal directivo, los docentes, los alumnos y las familias, quienes mantuvieron la comunicación a través de mensajería instantánea, llamadas telefónicas, videollamadas, correo electrónico y el perifoneo - esta última en las regiones de alta y muy alta marginación-. Con todo, la inclusión efectiva de las poblaciones indígenas, estudiantes con discapacidad, migrantes, escuelas comunitarias y, en general, los servicios educativos ubicados en contextos de pobreza constituyen el mayor reto (MEJOREDU, 2020a).

En la región, más del $40 \%$ de los docentes y los estudiantes se mantuvo en contacto a través de Google Classroom - una de las herramientas diseñadas por Google para la educación-; dato que colocó al Estado de Chihuahua en el cuarto lugar nacional en su acceso y en el primer estado en el país que desarrolló, en esta plataforma y con la colaboración del profesorado, sus propios materiales (Secretaría de Educación y Deporte (SEyD), 2020a). En la entidad, el servicio de mensajería y llamadas por WhatsApp representó la vía privile- 
giada en la comunicación entre docentes y estudiantes, convirtiéndose en "el gran salón de clases del Estado de Chihuahua” (SEyD, 2020a, 38m37s). Al uso del WhatsApp se sumaron llamadas telefónicas, documentos impresos, programas de televisión y radio (ver Tabla 1), lo que "significó que aproximadamente entre el $88 \%$ y el $92 \%$ del estudiantado mantuvo contacto con sus profesores" (SEyD, 2020a, 39m26s).

\section{Tabla 1}

Herramientas implementadas en la educación a distancia en Chihuahua

\begin{tabular}{ccc}
\hline Con Internet & Internet limitado o inexistente \\
\hline & TV Educativa & \\
& + & \\
& Radio (indí- & Material \\
Plataforma & gena) & impreso \\
Google para & + & $(380,000)$ \\
Educación & Correo elec- & \\
& trónico & \\
& & \\
& Telefonía y & \\
& WhatsApp \\
\hline
\end{tabular}

Nota: tomada de SEyD (2020b).

Entre los principales efectos de la pandemia destaca la modificación de los procesos de enseñanza y aprendizaje en todas las escuelas y todos los niveles del sistema educativo, particularmente, en los grupos de primer ciclo, donde:

los niños... en edad de aprender a leer y escribir, ... vieron modificados sus procesos de alfabetización. Lejos del aula y de sus amigos, con docentes reducidos a un rol de "acompañamiento" y padres empujados a ser docentes, los alumnos escriben y leen sus primeras palabras en un contexto de incertidumbre. (Brunetto, 2020)

En el escenario pandémico, los docentes se han enfrentado a diversos retos, sin embargo, para el profesorado de primer ciclo de educación primaria - que incluye los grados de primero y segundo- “el desafío es aún más significativo, porque enfrentan el reto de la alfabetización inicial en niñas y niños, lo cual implica fortalecer su práctica con estrategias didácticas, recursos y materiales que les apoyen en su trabajo" (MEJOREDU, 2020b, p. 4).

En el país, los procesos de lectura y escritura se desarrollan en la educación básica desde el componente curricular de Formación Académica que en la educación primaria refiere al campo formativo de Lengua Materna. Español, con la intención de fomentar entre el alumnado:

diversas prácticas sociales del lenguaje para fortalecer su participación en diferentes ámbitos, ampliar sus intereses culturales y resolver sus necesidades comunicativas. Particularmente busca que desarrollen su capacidad de expresarse oralmente y que se integren a la cultura escrita mediante la apropiación del sistema convencional de escritura y las experiencias de leer, interpretar y producir diversos tipos de textos. (SEP, 2017b, p. 163)

Ante el reto de aprender a leer y a escribir, la alfabetización va más allá de la codificación de letras y sonidos, implica conocer el código alfabético, dotarlo de significado y sentido para interactuar en los diversos contextos de la vida. Para Barton \& Hamilton (2000), la noción de alfabetización se distingue por seis rasgos: puede ser entendida como un conjunto de prácticas sociales mediadas por la escritura; está asociada a diferentes dominios de la vida; se configura en instituciones y relaciones de poder, por lo que algunas son más dominantes, visibles e influyentes que otras; emerge entre objetivos y prácticas culturales; está históricamente situada y se renueva en procesos de aprendizaje informal y construcción de sentido. Así, la alfabetización inicial constituye una herramienta fundamental para el aprendizaje y una condición en la solidez de las trayectorias académicas de las personas. 
Considerando que "la adquisición de la escritura requiere procedimientos especiales de enseñanza" (SEP, 2017a, p. 209), el presente artículo centra la atención en la intervención docente en los procesos de alfabetización inicial en tiempos de covid-19. Al respecto, Baez \& D’Ottavio (2020), Mairena \& Vijil (2020), Sánchez \& Santolària (2020) y Silva-Peña et al. (2016) coinciden en la importancia de las intervenciones del profesorado en los procesos de alfabetización inicial que, en general, "implica desarrollar en los niños la oralidad y favorecer su acercamiento a los usos sociales de la lectura y la escritura” (Guzmán, 2017, p. 107).

\section{Metodología}

El presente estudio se adscribe a la investigación cualitativa caracterizada por priorizar la comprensión de los significados que las personas, de forma individual o colectiva, atribuyen a un problema social o humano a partir de su propia experiencia (Creswell, 2014). En medio de la emergencia sanitaria, la investigación cualitativa posibilitó recuperar la voz de los docentes de educación primaria frente a la interrupción de las actividades escolares presenciales y la necesidad de enfrentar la alfabetización inicial a distancia. Se trata de un estudio de caso múltiple, de diseño holístico (Yin, 2003), en donde la experiencia de cada docente constituyó una situación o entidad social única (Sabariego et al., 2004) que, en conjunto, permitió comprender la complejidad del fenómeno educativo. Dado el carácter descriptivo del estudio, el ejercicio priorizó la interpretación de las experiencias docentes a la luz de las condiciones del contexto donde estas se configuran (Yin, 2003).

Así, el objetivo general del estudio fue recuperar la experiencia del profesorado de primer ciclo de educación primaria en torno a los saberes, las prácticas y las adecuaciones implicadas en los procesos de alfabetización inicial en tiempos de emergencia sanitaria. De este objetivo se derivaron tres específicos: a) indagar los saberes docentes considerados por el profesorado de primer ciclo de educación primaria en la alfabetización inicial en tiempos de pandemia; b) sistematizar las acciones y actividades implementadas por los docentes para dar continuidad a los procesos de alfabetización inicial a distancia; y c) documentar las adecuaciones docentes en la construcción de ambientes favorables para la alfabetización inicial durante la pandemia.

El estudio se desarrolló entre los meses de febrero y abril de 2021. La entrevista semiestructurada, técnica empleada para la recolección de datos, se implementó durante los meses de marzo y abril mediante la plataforma de videochat Google Meet, en atención a las restricciones planteadas por la sana distancia. Este tipo de herramientas constituye una valiosa oportunidad para enfrentar el desafío de la investigación cualitativa en tiempos de distanciamiento social (Lobe et al., 2020). La información se obtuvo con la colaboración de un grupo de 13 docentes ( 4 hombres y 9 mujeres) de primer ciclo de educación primaria, radicados en diversas regiones del estado de Chihuahua, localizado en el norte de México. Según las condiciones del contexto, Emilio, Hilda, Iris, Julia y Mary laboran en regiones con un grado de marginación/ rezago social muy alto y un nivel delictivo bajo; mientras, Amelia, Brenda, Carlos, Daniela, Flor, Gerardo, Kevin y Laura en regiones con un grado de marginación/rezago social muy bajo y un nivel delictivo alto (Secretaría de Bienestar, 2021). Los participantes fueron seleccionados con un muestreo intencional a partir de dos criterios: ser docente en activo y tener a su cargo el primer o segundo grado de educación primaria pública. Fueron determinados a través de un muestreo en cadena que permitió identificar casos de interés en personas que recomendaron a otras ricas en información (Miles \& Huberman, 1994 como se citó en Creswell, 2007). La cadena de referencia se cerró al alcanzar la saturación de los datos. En la Tabla 2 se caracteriza a los participantes bajo seudónimos a fin de proteger su identidad. 
Tabla 2

Caracterización de los participantes

\begin{tabular}{cccccccc}
\hline Clave & Sexo & Edad & $\begin{array}{c}\text { Experiencia } \\
\text { docente } \\
\text { (ańo) }\end{array}$ & $\begin{array}{c}\text { Número de } \\
\text { estudiantes }\end{array}$ & $\begin{array}{c}\text { Grado a } \\
\text { su cargo }\end{array}$ & $\begin{array}{c}\text { Lugar de } \\
\text { trabajo }\end{array}$ & Escolaridad \\
\hline Amelia & $\mathrm{M}$ & 32 & 7 & 35 & $2^{\text {o }}$ & Ciudad Juárez & Licenciatura \\
Brenda & $\mathrm{M}$ & 30 & 2 & 34 & $2^{\text {o }}$ & Ciudad Juárez & Licenciatura \\
Carlos & $\mathrm{H}$ & 25 & 3 & 19 & $1^{\text {o }}$ & Chihuahua & Licenciatura \\
Daniela & $\mathrm{M}$ & 30 & 5 & 34 & $1^{\text {o }}$ & Ciudad Juárez & Licenciatura \\
Emilio & $\mathrm{H}$ & 25 & 4 & 27 & $1^{\text {o }}$ & Coyame & Licenciatura \\
Flor & $\mathrm{M}$ & 28 & 5 & 34 & $1^{\text {o }}$ & Ciudad Juárez & Licenciatura \\
Gerardo & $\mathrm{H}$ & 23 & 1 & 27 & $1^{\text {o }}$ & Ciudad Juárez & Licenciatura \\
Hilda & $\mathrm{M}$ & 23 & 1 & 22 & $1^{\text {o }}$ & Las Varas & Licenciatura \\
Iris & $\mathrm{M}$ & 39 & 16 & 23 & $1^{\text {o }}$ & Palomas & Maestría \\
Julia & $\mathrm{M}$ & 26 & 4 & 14 & $2^{\text {o }}$ & Batopilas & Licenciatura \\
Kevin & $\mathrm{H}$ & 32 & 12 & 32 & $1^{\text {o }}$ & Delicias & Maestría \\
Laura & $\mathrm{M}$ & 22 & 1 & 23 & $1^{\text {o }}$ & Chihuahua & Licenciatura \\
Mary & $\mathrm{M}$ & 26 & 4 & 30 & $1^{\text {o }}$ & Coronado & Licenciatura \\
\hline
\end{tabular}

Respecto a las consideraciones éticas, la protección de los participantes en la investigación se realizó en apego a los siguientes principios: autonomía, se informó a los docentes de los propósitos del estudio mediante el protocolo de consentimiento informado; privacidad, participaron en la entrevista aquellos que aceptaron voluntariamente y bajo anonimato; paridad, se aceptó la participación de todos los informantes con la misma importancia; privacidad, se consideró clave la confidencialidad, el respeto a la intimidad y el derecho de la persona a elegir; y cautela en la emisión de juicios, se priorizó la objetividad en la descripción de las situaciones investigadas, evitando simplificar, exagerar u ocultar información (González et al., 2012).

Para el análisis de la información se recurrió a las tres estrategias propuestas por Creswell (2007): a) Revisión general de toda la información, luego de la transcripción de las entrevistas y la lectura - hori- zontal y vertical de cada caso y transversal del conjunto de casos-, se realizaron notas y reflexiones iniciales al margen; b) Reducción de la información, con la intención de condensar la información se elaboró una matriz de análisis cualitativo que permitió identificar contenidos recurrentes y relevantes alrededor de tres ejes temáticos (Sabariego et al., 2004); y c) Presentación de los resultados, para exponer los hallazgos se recurrió a la síntesis narrativa por cada uno de los ejes temáticos (ver Tabla 3). De este proceso se desprenden los resultados que se presentan a continuación. Dada la naturaleza de los datos se agregan citas textuales que ofrecen información detallada, densa y completa a fin de contribuir a la comprensión de la realidad mediante el análisis integrado y crítico de diferentes informantes - triangulación -; esto para cumplir con el criterio de credibilidad cualitativa (Creswell, 2007). 
Tabla 3

Ejes temáticos y categorias de análisis

\begin{tabular}{|c|c|c|c|c|c|}
\hline \multirow{4}{*}{ 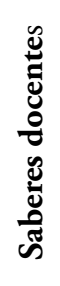 } & Formación docente & \multirow{4}{*}{ 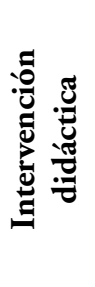 } & Situaciones didácticas & \multirow{4}{*}{ 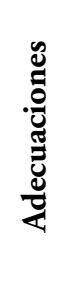 } & Experiencia \\
\hline & Procesos previos & & \multirow{2}{*}{ Recursos didácticos } & & Condiciones para el trabajo \\
\hline & Método de lectoescritura & & & & Ajustes \\
\hline & Motivaciones & & Evaluación & & $\begin{array}{l}\text { Preocupaciones ante el cierre del ciclo } \\
\text { escolar }\end{array}$ \\
\hline
\end{tabular}

\section{Resultados}

En este apartado se presentan los hallazgos en correspondencia a los objetivos que orientaron el estudio.

\section{Saberes docentes implicados en la alfabetiza- ción inicial}

El trabajo se realiza desde la noción de saberes docentes propuesta por Mercado (2018) para referir al "conocimiento que los maestros tienen sobre la enseñanza y que desarrollan durante el ejercicio cotidiano de la docencia" (p. 11). En el discurso docente, los saberes para la enseñanza se adquieren en la formación inicial, sin embargo, es en el trabajo cotidiano donde "se aprende en realidad a ser maestro" (p. 165).

Frente a la experiencia de la alfabetización inicial en la contingencia, los docentes valoran su formación docente inicial frente a tres desafíos: la práctica docente, la educación a distancia y la enseńanza de la lectoescritura. El primer desafío emerge probablemente porque cinco de los docentes son noveles, enfrentándose a la inserción profesional entendida como "un periodo de tensiones y aprendizajes intensivos en contextos generalmente desconocidos y durante el cual los profesores principiantes deben adquirir conocimiento profesional" (Marcelo, 2008, p. 14).

[Como docente de] nuevo ingreso uno entra con muchas dudas, con muchos miedos (Brenda).

Siento que mi formación docente fue insuficiente para manejar un grupo de primer año. La primera vez casi sali llorando porque no sabia cómo trabajar con los niños (Flor).

Fue suficiente..., pero estamos en [una profesión en] constante cambio; hay que seguir investigando para enfrentar cualquier situación (Laura).

Ante los efectos de la pandemia por covid-19, el cierre de las escuelas y la implementación de acciones de educación a distancia fueron inminentes. Para el profesorado, la experiencia develó debilidad en las competencias digitales necesarias para atender los requerimientos de la propuesta gubernamental que requería, por ejemplo, el manejo de procesadores de texto, hojas de cálculo, presentaciones, correo electrónico, elaboración de videos y juegos interactivos, reuniones por videollamada, entre otros. En respuesta, los docentes recurrieron al Internet y a sus pares para resolver dudas, intercambiar ideas y compartir información.

Fue más autodidacta; tuvimos que buscar y trabajar en conjunto y ver qué herramientas eran útiles $y$ compartirnos información. [Aprendimos], sobre la marcha (Daniela).

Me considero un maestro aún en formación... Estoy en constante aprendizaje, he revisado muchas estrategias para usar las plataformas, las herramientas, $y$ las diferentes aplicaciones (Gerardo).

Ha sido un gran reto... No estamos familiarizados con la educación vía remota. Ha sido complicado tanto para alumnos, padres de familia como para nosotros debido a que, en ocasiones, no contamos con los medios, la capacitación, los conocimientos que 
implica todo esto; además, también ha sido complicado hacer las modificaciones para que las actividades sean más sencillas, dinámicas y fáciles, tanto para que los padres de familia las puedan entender y transmitir a sus hijos, como para que a los alumnos no les resulten complicadas (Iris).

El tercer desafío alude a la responsabilidad de atender a un grupo de primer ciclo de educación primaria, en especial, porque uno de sus propósitos refiere a que los estudiantes avancen en su conocimiento del lenguaje oral y escrito.

[Ser] docente de primer grado es una responsabilidad porque es un año crucial... al principio me dio un poco de incertidumbre, ¿cómo iba a lograr que los niños en realidad llegaran ese nivel? (Emilio).

Ha sido un reto. La verdad al inicio pensaba que se me hacía un mundo y no sé cómo le voy a hacer, qué actividades y cómo vamos a trabajar (Daniela).

Es la primera vez que tengo primer grado y pensé ¿qué voy a hacer? Asi a distancia es mucho más complicado (Mary).

Dado que la adquisición del lenguaje escrito "no depende de la copia ni de la producción repetida de textos sin sentido" (SEP, 2017b, p. 168), según lo declarado por los docentes, el aprendizaje de la lectura y la escritura conlleva tiempo y requiere de situaciones didácticas específicas para acercar a los estudiantes a la alfabetización inicial.

De agosto a octubre no utilizo los libros de texto... [Busco], cuando menos, quince o veinte minutos de lectura diaria; de libros que estén adecuados a su edad biológica, psicológica y cognitiva (Amelia).

Es un proceso largo de dos años. Lo primero es el contacto de los alumnos con el dictado... Después, el cuento. [También trabajamos con] las características de un buen alfabeto (Brenda).

Primero se trabaja con los niños en su motricidad fina y gruesa... Incluso fortalecer cómo agarrar el lápiz, entonces di muchas indicaciones a los papás en este tiempo. No iniciamos de lleno a trabajar con las letras, primero con juegos y portadores de texto (Kevin).

Entre el profesorado se reconoce la importancia de la educación preescolar. Los alumnos que ingresan a la educación primaria, luego de un periodo de escolarización previo, se distinguen por sus relaciones con otros niños y adultos, por estar familiarizados con la lectura y la escritura en un nivel inicial y, en el caso particular, por conocer la dinámica del trabajo a distancia.

Por el historial que traen del preescolar siento que me he enfocado con los alumnos con más deficiencias de aprendizaje (Iris).

Primero me dediqué a conocer a los niños, qué es lo que ellos sabian. Como llegaron del preescolar conocian las letras y los números (Mary).

Frente a la alfabetización inicial, un aspecto clave refiere a la intervención docente para generar prácticas de lectura y escritura (Lerner, 2001). Posicionarse frente a un tipo de intervención didáctica $\mathrm{u}$ otro es un tema de debate entre el profesorado, entre otras cosas, por la prontitud de sus resultados o sus implicaciones en la comprensión lectora. Si bien el empleo de los métodos para la enseńanza de la lectura y la escritura han dejado de ser una prioridad para la investigación educativa actual, su uso sigue vigente en las preocupaciones del profesorado (Guzmán, 2017). En la experiencia declarada por algunos docentes se encontró una justificación instrumentalista en el uso de los métodos de lectoescritura que, como señala Guzmán, se centra en atender pasos de modo prescriptivo y mecánico, sin considerar las características de los alumnos y sus contextos. Al respecto, Lerner (2001) invita a considerar los avances del conocimiento pedagógico para "mostrar los efectos nocivos de los métodos y procedimientos tradicionales que resultan tan 'tranquilizadores' para la comunidad, y hacer públicas las ventajas de las estrategias didácticas que realmente contribuyen a formar lectores y escritores autónomos" (p. 66). 
Se parte de la palabra completa, por ejemplo, se les dicta - hay niveles de dictado-, entonces nos piden que entre más dificultosas sean las palabras es mucho mejor para los niños... Nos dieron un alfabeto viable para enseñar por el sonido de la letra y explicarles que tienen también un nombre (Brenda).

Estoy usando el método alfabético... [mediante] un cuadernillo donde vienen todas las letras del abecedario y en cada letra vienen actividades propias de la letra y una lectura pequeña. [Además] es el más fácil para que los papás en casa también se pongan con ellos a repasar (Hilda).

En algunos casos, el limitado conocimiento sobre los métodos se diluye en afirmaciones difusas que aluden a los beneficios de combinar métodos para mejorar los resultados.

Estoy utilizando una metodología que viene dentro de esta reforma educativa, en el último programa, en realidad no sé ni cómo se llama (Emilio).

La maestra de $1^{\circ} B$ y yo estamos trabajando igual... Estamos haciendo una mezcla —un Frankensteinde lo que se pueda, porque la mayoría de los papás enseñan como ellos aprendian (Flor).

No tengo un método especifico... Trabajo mucho la alfabetización a través del dictado y el repaso de sonidos, de letras, la relación sonoro-gráfico y también imagen-texto, el alfabeto móvil, la rima, las palabras generadoras, los portadores... he tomado un poquito de todos los que existen (Iris).

Para algunos docentes el uso de un determinado método es resultado de la indicación directa de la dirección o supervisión escolar o la propuesta señalada en algún curso organizado por las autoridades escolares.

El método que nos piden trabajar en nuestra zona escolar es el psicolingüistico (Amelia).

El PRONALEES fue una recomendación de la inspección escolar. Los niños avanzan a su propio ritmo... [en] la parte de la lectura, sinceramente leen muy bonito, en comparación de cuando se les enseña con el método silábico (Brenda).
Por parte de supervisión nos dieron un taller de alfabetización, pues somos muchos maestros noveles. El supervisor nos enseñó las teorias de cada tipo de enseñanza, los métodos que existen, tips de vocalización, de motricidad fina (Gerardo).

En otros casos, la elección de la modalidad de intervención refiere a iniciativas impulsadas por la SEP en diferentes momentos de la historia en el país que se mantienen en la memoria magisterial como prácticas exitosas; por ejemplo, la Propuesta para la Adquisición de la Lengua Escrita y las Matemáticas (PALEM) que se promovió de 1990 hasta 1997 y el Programa Nacional para el Fortalecimiento de la Lectura y la Escritura en la Educación Básica (PRONALEES) que estuvo vigente hasta 2002. Ambas iniciativas se sustentaban en el enfoque comunicativo y funcional de la alfabetización "para un uso efectivo del lenguaje escrito en diversas situaciones y propósitos comunicativos de la vida cotidiana” (Espinosa \& Mercado, 2008, p. 204).

\section{[Utilizo] el que sugiere PRONALEES (Brenda).}

No tengo un método especifico. Utilizo un poco de todo: el juego, incluso las fichas del PALEM, que es un método que se utilizó en la década de los 90, pero sigue siendo muy funcional (Kevin).

Además, las motivaciones de la elección se hacen considerando tres aspectos: su efectividad, su contribución a la comprensión lectora y la facilidad para el trabajo en casa. Una exigencia se refiere a la consolidación de la lectura y la escritura. De acuerdo con la normativa oficial, se espera que los nińos lean y escriban "de manera convencional y autónoma... hacia el segundo grado de educación primaria" (SEP, 2017b, p. 167), por lo que la presión de las familias, las autoridades escolares y de los propios docentes representa un factor clave para seleccionar modos de intervención que ofrezcan resultados a corto plazo. Otros docentes consideran las bases que estos ofrecen para la comprensión de la lectura, en especial, porque este punto constituye una dificultad educativa en el país. "El problema radica en el inicio de la formación de 
los niños como lectores... Pareciera ser que, entre más pronto mejor, y la mejor forma de hacerlo rápido se halla en lo superficial del lenguaje" (Aguilera, 2020).

Como es un grupo pequeño me fui con el silábico, para mi fue muy funcional. Ya al último fue el global porque es más fácil y práctico (Julia).

Estamos utilizando mayormente silábico -que no me gusta porque los niños pierden mucho en lo que es comprensión - pero como nos están pidiendo resultados... Siento que nos piden que simulemos, que estamos haciendo el trabajo [pero] cuando regresemos, van a evaluarlos y van a ver que, en realidad, hay un retraso enorme. $Y$, ¿quién va a ser el culpable? Pues voy a ser yo, porque yo soy la docente (Flor).

La tercera razón alude a las condiciones derivadas por la pandemia. Para dar continuidad a los procesos de enseńanza y aprendizaje, las madres y los padres de familia se encargaron de orientar a sus hijos en la resolución de las tareas escolares. A nivel estatal, "la madre de familia fue la maestra alterna en un $90 \%$ de los casos" (SEyD, 2020, 57m27s). De acuerdo con lo declarado por los docentes, la mayoría de las familias respondió a la invitación para continuar con el trabajo escolar en casa, a pesar de las carencias económicas y tecnológicas, a las que se sumaron los casos de madres y padres que "no tienen estudios, algunas de ellas no saben ni leer ni escribir" (Mary). Así, en la elección del método didáctico se consideraron aquellos que resultaran sencillos para los integrantes de las familias, encargados de acompañar a los alumnos en el proceso.

Lo escogi por comodidad para los padres de familia (Hilda).

Le tienes que explicar al papá hasta el cronograma, ya que es como el maestro en casa. Considero que, si los niños han leido, es gracias al diseño de cuadernillos y al apoyo de los padres (Julia).

La interacción con los niños ha sido poca; la verdad con quien más he trabajado es con los padres de familia (Mary).
Dada la importancia del apoyo de las familias, los docentes señalan prioritario capacitarles en diversas temáticas para el trabajo a distancia, lo que implicó sensibilizarles ante la necesidad de sumar esfuerzos frente a la emergencia sanitaria, orientarles en el uso de plataformas digitales, facilitarles materiales, explicarles el método de lectoescritura, reconocer continuamente el valor de su colaboración y ofrecerles actividades que permitieran la convivencia familiar. En este punto, destaca la presencia de diferentes figuras familiares, como los hermanos mayores y las abuelas, sobre quienes, en algunos casos, recayó el acompañamiento de los estudiantes en casa.

Al principio estaban muy molestos e inconformes... No querian trabajar en linea. Entonces, lo principal fue realizar un trabajo de convencimiento entre la subdirectora y yo para que los padres se dieran cuenta de que al final el beneficio era para sus hijos. Si fue muy complicado, pero también fue trabajar con mucha empatia... comprender y decirles que entendiamos su situación porque muchos de ellos empezaron a quedarse sin empleo (Amelia).

Ayudé a los padres sobre cómo usar Classroom, cómo usar la plataforma, cómo subir las evidencias... Les fui aconsejando sobre cómo utilizar el correo institucional (Carlos).

En el grupo ya estaban un tanto familiarizados con las clases en línea porque a lo mejor tienen hijos más grandes en la universidad o en la secundaria, asi que no batallé. Tengo una abuelita con dificultades para usar el WhatsApp, lo que hago es que le mando los trabajos a una papelería y ella va y lo recoge; luego, cada 15 dias voy por los trabajos y me los traigo y le digo Ahi hay más trabajo para que vaya por ellos (Hilda).

Me tocó ayudar a algunos a descargar WhatsApp, conseguirles en la biblioteca de la comunidad la clave del Internet de la escuela. Luego decirles cómo iba a ser la dinámica de las videollamadas porque a veces no respondian (Iris).

Entre los métodos señalados por los participantes destaca el método global de análisis estructural, 
implementado en México en la década de los setenta, que se caracteriza por propiciar la maduración, enseñar a leer y escribir por estructuras de significado, así como por favorecer la comprensión lectora (Galicia, 1978).

Apliqué todos, hasta que me di cuenta de que el método global es muy bueno. Entonces trabajo el método global por campos semánticos; por ejemplo, trabajo con su núcleo familiar y eso me ha servido bastante para incluir a los niños con discapacidad, porque son palabras que se identifican. [Con ellos] trabajo el método Troncoso (Amelia).

Empecé con el silábico, el tradicional que yo conocía. Después tomé un curso sobre el método global, se me hizo muy padre y hasta la fecha lo sigo aplicando (Laura).

El método global lo empezamos a trabajar porque veíamos que funcionaba con los alumnos (Daniela).

\section{Intervención didáctica para la alfabetización digital}

En el modelo vigente, los maestros son reconocidos como pieza clave en el éxito de los cambios educativos. Entre las características de un buen docente destaca su capacidad para organizar el trabajo pedagógico y realizar "una intervención didáctica pertinente" (SEP, 2017b, p. 41). La pertinencia refiere a la correspondencia entre las situaciones didácticas y las características del "contexto, así como en las necesidades educativas y los intereses de los estudiantes (p. 374).

Las situaciones didácticas son el eje del trabajo en el aula - física o virtual-. En ellas se organizan las tareas y se prevén los materiales en torno a determinados contenidos para promover el aprendizaje de acuerdo con los distintos contextos, niveles y necesidades particulares de los estudiantes. En la narrativa docente, la mayoría de las situaciones didácticas se vinculan al desarrollo de las prácticas sociales del lenguaje en el ámbito de Literatura, dirigidas a que los estudiantes "comprendan la intención creativa del lenguaje, amplíen sus horizontes culturales y aprendan a valorar diversos modos de comprender el mundo y de expresarlo" (SEP, 2017b, p. 174).

Les reparto a mis alumnos cuentitos y libros muy sencillos (Amelia).

El cuento es lo primero que hacemos y luego el dictado (Brenda).

Tengo cuentos, en realidad son audiolibros, para que los alumnos los escuchen y se resaltan sonidos de las grafias (Iris).

Otras situaciones didácticas se relacionan con las prácticas sociales del lenguaje en el ámbito de Participación social que tienen como intención "desarrollar y favorecer las maneras de participar en la construcción de la sociedad [mediante] la producción e interpretación de textos de uso cotidiano en ambientes alfabetizados vinculados con la vida social" (SEP, 2017, p. 175).

Justo en la esquina de la escuela vive Ana —una estudiante con discapacidad motriz-. Alli pusimos un buzón en febrero con el motivo de San Valentín, pero vimos que la actividad funcionó tan bien que lo dejamos como actividad permanente en el grupo. Los niños van y dejan cartas para sus amigos. Una vez por semana van y las dejan, luego Ana es la encargada de cuidar el buzón (Amelia).

En el discurso docente aparece el juego como elemento que impulsa el aprendizaje de la lectura y la escritura a través de canciones, rimas y trabalenguas que por su carácter lúdico resultan divertidas; en la pandemia, mantener a los niños frente a la pantalla constituyó un nuevo reto. Para atenderlo, los docentes renovaron los materiales didácticos tradicionales incorporando elementos audiovisuales, elaboraron o descargaron videos, juegos interactivos, canciones con letra, entre otros recursos digitales.

Lecturas regaladas a los alumnos — tres maestras les hacíamos un videito con tres cuentitos-, de eso les preguntábamos. Luego ellos expresaban qué entendieron, qué les gustó o qué no les gustó (Daniela). 
A mi me gustaba mucho implementar el karaoke, como el de Gallinita Pinta (Julia).

He utilizado mucho las diapositivas animadas... les pongo imágenes y sonidos que llamen la atención de los niños (Kevin).

Con el propósito de favorecer la alfabetización, los docentes emplearon una serie de materiales didácticos de diseńo y recursos propios, de autoría colectiva, disponibles en la red, así como materiales educativos impresos y digitales, elaborados por las autoridades educativas locales o la SEP. En el caso de los cuadernillos de ejercicios, se distinguen dos tipos: aquellos elaborados por las instancias oficiales y de distribución masiva, y aquellos elaborados por los propios docentes y de distribución limitada. En algunos casos, los materiales se entregaron de forma física y sin costo para las familias, en otros se compartieron en archivo electrónico, dejando a consideración de los padres su impresión. Pese a la frecuencia de su empleo, los materiales impresos tienen la desventaja de limitar las interacciones sociales necesarias para la construcción del conocimiento, particularmente, para hacer de cada actividad "un desafío para los niños en proceso de alfabetización” (Hoz et al., 2021, p. 291).

Tenemos cuadernillos fotocopiados... me di a la tarea de realizar diferentes actividades para completar, remarcar, repasar, leer imágenes, el dictado diario y el alfabeto móvil (Iris).

Trato de enviarles cuadernillos donde ya venga el ejercicio como tal, porque para los papás es más práctico que escribir la actividad y que el niño la haga (Laura).

Las referencias a los materiales oficiales por parte de los docentes fueron mínimas. El uso del libro de texto gratuito aparece como una actividad complementaria, probablemente porque la entrega fue tardía; mientras, el empleo del Programa Aprende en Casa por televisión solo fue señalado por algunos docentes, particularmente, por aquellos que laboran en comunidades donde el acceso a Internet es insuficiente o inexistente.
No dejo de trabajar el libro de texto porque también está encaminado a la adquisición de la lectoescritura. No es muy dinámico, pero es de gran ayuda (Iris).

Voy trabajando conforme vienen los aprendizajes que marcan los programas de la televisión (Mary).

En la enseńanza de la lectura y la escritura, la evaluación es un componente necesario para valorar el logro de los aprendizajes, identificar las dificultades, tomar decisiones e implementar estrategias diferenciadas a partir de la recolección, sistematización y análisis de "información obtenida de diversas fuentes derivadas del trabajo, en distintos momentos, con las prácticas sociales del lenguaje” (SEP, 2017b, p. 182).

Frente al tema de la evaluación, la experiencia docente gira en torno a tres preguntas: qué, cuándo y cómo evaluar.

Según los docentes, la evaluación, más que centrarse en quién lee y escribe de modo convencional, recupera las producciones — en forma de evidenciasque dan cuenta de los avances de los niños durante el proceso, en apego a la evaluación formativa; así como del uso de diversas herramientas para documentar las valoraciones por período.

Hago una rúbrica donde hago una jerarquización de los aprendizajes que ellos tienen que lograr (Amelia).

Se tomó el acuerdo de utilizar un formato de Google Classroom que permite obtener resultados rápidamente... las evidencias 70\%, autoevaluación 10\% de la calificación —no se toman de criterio las asistencias, porque a algunos papás se les complica mucho la conexión-(Carlos).

Les llevo un registro en Excel de las actividades de lectoescritura, matemáticas... También evalúo participación, asistencia y videollamadas —en ocasiones para ver cómo van avanzando, tomarles una lectura o dictado-(Daniela).

Respecto a los momentos para evaluar, los docentes aluden a la evaluación inicial con la intención de identificar los conocimientos previos o las concepciones 
que los estudiantes tienen sobre los componentes de la práctica social del lenguaje; a la evaluación permanente para valorar lo realizado y aprendido durante el proceso, y a la evaluación final, en correspondencia al logro de los aprendizajes esperados.

Antes de iniciar con el proceso de alfabetización se realizó un diagnóstico, no como se hace en forma presencial, pero que permitiera saber en qué nivel se encontraban los niños. También la revisión del historial de preescolar fue importante (Iris).

Cada semana registro las evidencias... [Además] trato de realizar la reflexión tanto de mi trabajo como del de ellos (Laura).

[Evalúo] por medio de los trabajos que me van enviando los padres de familia. A veces al terminarse el trimestre y quienes me envian diariamente las actividades. También hay muchos que no mandan nada (Mary).

La evaluación la llevo cada 15 días con los silábicos y los alfabéticos, con los presilábicos por semana ... Me preocupa que los niños no estén listos para pasar a segundo grado, se van a topar con aprendizajes que no se concretaron (Flor).

Ante la necesidad de continuar con los procesos educativos en medio de las restricciones de movilidad y contacto social, los docentes refieren a distintas estrategias para llevar a cabo la evaluación de los aprendizajes adquiridos por los estudiantes en casa. Según Lerner (2020), pese al esfuerzo del profesorado, resulta difícil retroalimentar al estudiantado, sobre todo, por el tamaño de los grupos y el tipo de actividades, generalmente, individuales.

Me dediqué a hacer un calendario en el cual decía este día puedo llamar a Cristal, este dia a María y en este horario a Daniel... Les hacía videollamadas por WhatsApp... Los llamo directamente si es una videollamada necesaria y, en ocasiones, cuando existe la posibilidad, voy a sus casas (Amelia).

Este trimestre quise hacer una pequeña evaluación a través de una videollamada y no me resultó... tuve que optar — con la autorización de mi autoridad inmediata superior - por citar, en horarios diferentes, a los niños y con las medidas pertinentes, para realizar una evaluación presencial de lectura, escritura y operaciones básicas (Iris).

\section{Adecuaciones ante la emergencia sanitaria}

La pandemia generó un cambio brusco en las condiciones del trabajo docente que "llevó a problematizar formas de enseñanza que estaban naturalizadas [ante lo cual]... los maestros tuvimos que reinventarnos" (Lerner, 2020, 14m51s). Para el profesorado, la experiencia ha significado un período de incertidumbre valorado como difícil y estresante que puede interpretarse desde el ámbito personal y profesional.

En un principio fue difícil. Cuando inició la pandemia entré en estrés, porque no solamente era la cuestión de cómo hacer llegar la información a los alumnos, sino cómo ayudarle a los papás; luego existió la parte administrativa por parte de dirección... nos saturaron en cuestión de evidencias (Brenda).

Muy frustrante... Nos piden que seamos empáticos con los padres de familia, que no les dejemos tantas actividades..., pero al momento de las evaluaciones quieren que existan buenos resultados (Flor).

Muy difícil, la verdad. Es algo muy complejo y complicado. Se necesita de mucho apoyo por parte de nuestra plantilla docente, de nuestro director y de los padres de familia (Laura).

Entre los obstáculos en el desarrollo de las actividades se identificaron cuatro situaciones: personales, formativas, contextuales y de atención a la vulnerabilidad. Respecto a las dificultades de tipo personal, el profesorado cuenta con computadora particular y servicio de Internet; sin embargo, Brenda, Flor, Iris y Mary carecen de un espacio para el trabajo en línea; sobre las limitaciones de tipo formativo, Amelia, Brenda y Julia consideran insuficientes sus competencias para el trabajo a distancia. 
Organicé mi espacio de enseñanza, un espacio para atender mejor al grupo (Carlos).

Contaba con Internet y con computadora, pero tuve que adecuar un espacio para trabajar (Mary).

La primera es mi ignorancia respecto a ciertos dispositivos, por ejemplo, como utilizar WhatsApp al 100\%, Google Meet, Classroom y todas las diferentes aplicaciones posibles, pero me he estado capacitando (Gerardo).

Entre los inconvenientes se señalan las condiciones del contexto, en específico, la pobreza, el nivel cultural de las familias, la falta de recursos tecnológicos y acceso a internet o el alto costo de los planes de prepago para esto, que se agravan según la región donde los docentes laboran.

[La escuela está en] una zona marginal donde hay mucha violencia y carencias de agua, luz, drenaje... De los veintitantos niños con los que tenía comunicación, cuatro tenian internet, asi que no consideré las clases en linea. Entonces pensé en un grupo de WhatsApp que funcionó al principio hasta que se les saturaba el teléfono de tantas fotos; asi opté por hacer un grupo en Facebook y ahi subir las actividades para que los padres se comunicaran cuando tuvieran oportunidad de poner saldo, porque me explicaban que se les acababan los datos y tenían que esperar hasta el jueves que les pagaran (Brenda).

La mayoría de los niños cuentan con equipo tecnológico, computadoras o bien el celular de sus papás (Kevin).

Trabajo en una zona rural y muchos niños no tienen cómo comunicarse, no tienen Internet, no tienen tanta facilidad para hacerles llegar el trabajo (Mary).

A las dificultades anteriores se agrega la atención a estudiantes en condición de vulnerabilidad. En los relatos del profesorado se distingue la presencia de alumnos con discapacidad motriz, visual e intelectual; no obstante, las referencias a adecuaciones al trabajo escolar son mínimas, lo que confirma que atender a la distancia a los estudiantes con barreras para el aprendizaje, en especial, a aquellos de comunidades en zonas "de muy alta y alta marginación, representó un reto de enormes magnitudes, no solo para acogerlos en contexto de emergencia, sino para evitar que se acentuaran aún más los problemas de rezago educativo" (MEJOREDU, 2020a, p. 75).

En el grupo hay niños que tienen discapacidad visual y auditiva (Amelia).

En el grupo hay dos alumnos con [Trastorno por Déficit de Atención] TDA (Carlos).

Tengo una niña con sindrome de Down (Flor).

Tengo un alumno que tiene autismo (Hilda).

Pese a las iniciativas gubernamentales, escolares y docentes, algunos estudiantes han quedado excluidos de los servicios educativos durante la contingencia. De acuerdo con los relatos del profesorado, en la mayoría de los casos se ha perdido el contacto con, por lo menos, dos estudiantes por grupo. A nivel nacional, las causas de la exclusión se encuentran en "la imposibilidad de mantener comunicación con sus escuelas, la falta de dispositivos tecnológicos como computadora o internet..., la necesidad de atender labores del hogar, cuidar de otras personas, y la desmotivación” (MEJOREDU, 2020a, p. 58), a las que se suma la población flotante en la región.

Tengo 34 alumnos... de ellos tengo tres con quienes no tengo comunicación absoluta (Daniela).

De los 22 niños, con dos no he tenido comunicación porque de plano no sé ni siquiera dónde viven, ni tengo su número de teléfono (Hilda).

De los 23 con 21 be tenido comunicación; con los otros dos no sé nada... están totalmente perdidos (Laura).

Para enfrentar los desafíos impuestos por la pandemia, los docentes tuvieron que ajustar el trabajo escolar para su implementación a distancia, entre los principales cambios se encuentran: la flexibilización 
de las actividades, el diseño de materiales educativos, el empleo de herramientas digitales y el establecimiento de redes de apoyo entre docentes.

Los padres empezaron a decirme que era demasiado. [Les dije], saben que comprendo totalmente... [asi] la carga de materias y actividades se redujo al minimo (Amelia).

Eliminé esa parte [imprimir material], ¿qué me quedaba? O hacer juegos con material que tuvieran las mamás o nada más trabajar con el libro, ahorita es solo trabajar con el libro (Emilio).

Uso de herramientas digitales, resumir y simplificar mucho las actividades, explicaciones breves y claras con los papás (Gerardo).

El "Uber homework" ... una maestra tuvo la idea y nos dijo... ¿qué les parece si voy y reparto? Yo le envio el material por WhatsApp, ella lo imprime y me hace el favor de entregarlo (Laura).

Ante el cierre del ciclo escolar, el profesorado reconoce preocupación por el rezago educativo, en especial, de aquellos estudiantes excluidos, la persistencia de la pandemia y, particularmente, la atención socioemocional a los estudiantes ante las pérdidas humanas y el duelo.

Mi única preocupación es si realmente mis alumnos están preparados para el siguiente nivel... cuando lleguemos creo que vamos a tener bastante rezago ¡Si ya habia!, pues ahora va a haber muchisimo más (Kevin).

Los alumnos intermitentes e inexistentes (Daniela).

Que las medidas no sean suficientes y tengamos que volver a salir de la escuela, me preocupan los contagios (Gerardo).

Tengo seis niños sin mamá, ya porque su mamá murió por covid-19, y sin abuelos, sin tíos... Ha sido muy complicado para ellos (Amelia).

En el balance, el profesorado reconoce que la pandemia no solo significa incertidumbre y dificultad, sino una oportunidad para fortalecer sus competencias digitales, establecer relaciones de empatía y trabajo conjunto con las familias, conocer en profundidad a los estudiantes y contar con el reconocimiento de la sociedad a su trabajo.

La relación con papás y alumnos en conjunto... Nos dimos cuenta de qué es lo que está pasando en sus casas... Aprender a utilizar tecnologías, desarrollamos habilidades y construimos una mejor comunicación (Gerardo).

Estar con los alumnos... en esas situaciones que a veces en la escuela son muy difíciles de apoyar... En casa muchas veces hay historias de fondo muy dificiles (Laura).

Los padres de familia ahora se dieron cuenta de lo importante de nuestra tarea (Kevin).

\section{Conclusiones y discusiones}

En la experiencia concreta, el profesorado puso en juego una serie de saberes docentes que reconstruyeron en la práctica y en la interacción con otros - docentes, directivos, estudiantes y familias-. Para algunos docentes, la experiencia mostró la fragilidad de su formación y la necesidad de apelar a conocimientos de distinta índole, a veces contradictorios entre sí; para otros, permitió movilizar los saberes, incentivados en la formación inicial, y fortalecerlos a partir de su propia iniciativa, el trabajo colectivo entre pares y la solidaridad de las familias, con la finalidad de afrontar los problemas, superar las carencias y actuar en favor de los nińos. Al respecto se coincide con Mercado (2018), para quien las acciones que los docentes emprenden son resultado tanto del trabajo individual como colectivo y del conocimiento que estos tienen sobre sus alumnos, de sus logros, dificultades e intereses.

Para el profesorado, atender la alfabetización inicial en la pandemia requirió de la puesta en práctica de saberes de diversa índole: didáctica, para el diseńo de situaciones de aprendizaje — vinculada con el saber docente de la planeación o "anticipación de la ense- 
ñanza" (Mercado, 2018, p. 84), que usualmente se realiza entre dos tensiones: la planeación oficial y la no-escrita que se amplía, sustituye, complementa o improvisa mientras transcurre la enseñanza; teórico-metodológica, para impulsar prácticas de lectura y escritura; interpersonal, para el establecimiento de relaciones de colaboración con docentes, estudiantes y familias; evaluativa, para valorar los logros del alumnado y ajustar el proceso; digital, para hacer un uso innovador de las Tecnologías de la Información y la Comunicación (TIC), a fin de promover el aprendizaje y su propio desarrollo profesional. La experiencia local confirma que los docentes "ante una situación emergente e inédita... buscaron generar las condiciones más adecuadas para enfrentar las necesidades surgidas a partir de diversos procesos de ajuste, innovación y adaptación” (MEJOREDU, 2020a, p. 73).

Para la alfabetización inicial a distancia, el profesorado configuró una intervención didáctica entre situaciones de aprendizaje vinculadas al desarrollo de las prácticas sociales del lenguaje, principalmente, en los ámbitos de la literatura y la participación social; el juego, con la intención de retener la atención del estudiantado durante las sesiones virtuales o llamadas telefónicas; un repertorio de recursos didácticos desde los tradicionales - material concreto, cuadernillos de trabajo, libretas, libros de texto y cuentos-, hasta los emergentes — audios, videos, plataformas digitales, videollamadas, televisión y redes sociales; donde las referencias a los materiales oficiales fueron mínimas. Para Mercado (2018), este es un tipo de saber docente que refiere "a la búsqueda, uso y combinación de apoyos que los maestros consideran pertinentes para la enseñanza” (p. 84). Los resultados coinciden con Baez \& D’Ottavio (2020), Lerner (2020) y Sánchez \& Santolària (2020) acerca de la importancia de la intervención docente en los procesos de alfabetización.

Ante la emergencia sanitaria, los docentes tuvieron que adecuar el trabajo escolar, implementar una serie de cambios y enfrentar la incertidumbre. La experiencia, valorada inicialmente en términos de dificultad, estrés y carencia, se convirtió en forma gradual en la posibilidad para desarrollar nuevas competen- cias digitales, interpersonales, socioemocionales, entre otras. Con todo, el profesorado mantiene un sentimiento de deuda ante los niños, sus familias y frente a sí mismos que puede explicarse como resultado de las políticas educativas de corte neoliberal a favor de "la precarización del trabajo docente y la emergencia de nuevas estrategias de control, basadas en la auditoría, en el desempeńo y en el recrudecimiento de la culpa y de la auto-responsabilización docentes" (Alves \& Barreto, 2006, p. 184).

En la narrativa docente, las TIC aparecen de manera recurrente en forma de reto, solución, debilidad formativa, apoyo, aspiración, entre otras. Para atender los desafíos de la educación a distancia, el uso de las TIC representó un vacío en la formación del profesorado; en ese sentido, los apoyos gubernamentales y escolares se centraron en la capacitación. El desarrollo de las competencias docentes en el ámbito de las TIC remite a un tema de interés, previo y posterior a la pandemia, que requiere de trayectos formativos orientados a superar el uso puramente instrumental de las TIC para guiar a los docentes en la adquisición, profundización y creación de conocimientos de las tecnologías en materia educativa (UNESCO, 2019).

Las prácticas docentes variaron debido a la zona geográfica y socioeconómica del alumnado; sin embargo, fue común el uso de WhatsApp para enviar mensajes, imágenes y audios, hacer llamadas y videollamadas; además, su empleo develó la reducción del papel de los estudiantes a hacedores de tareas en lugar de lectores y escritores activos (Chamberlain et al., 2020). El uso de esta herramienta destaca en otros estudios como la vía privilegiada para la comunicación entre los actores educativos (MEJOREDU, 2020a), a pesar de sus desventajas, como la interacción asincrónica, la necesidad de datos móviles o conexión a Internet, y la mediación de la familia como destinatario indirecto (Hoz et al., 2021). Al margen de los servicios educativos, quedaron excluidos algunos estudiantes, particularmente, aquellos en situación de vulnerabilidad. El estudio confirmó, como señalan Forteza-Forteza et al. (2021) y Zepeda (2020), que la pandemia generó 
dificultades adicionales para el aprendizaje de los estudiantes con discapacidad, quienes enfrentan invisibilidad educativa. Ante la heterogeneidad del alumnado, el profesorado requiere "implementar estrategias que promuevan que los grupos se nivelen sin que ningún alumno deje de aprender" (SEP, 2017b, p. 69), en forma de ajustes razonables y recursos educativos adicionales en su atención.

A partir del análisis, la formación docente se resignifica a la luz de los resultados sobre las intervenciones de los docentes en la enseñanza de la lectura y la escritura, así como las razones declaradas en su elección. En este punto se coincide con Guzmán (2017), quien encontró confusión en los argumentos del profesorado sobre los métodos utilizados: "se refieren a ellos con nombres específicos..., pero no hay ninguna alusión a sus postulados epistemológicos ni a sus lógicas internas, ni se refieren de manera explícita a lo que implica el manejo de cada uno de ellos" (p. 109). Así, no solo son necesarias estrategias formativas para atender las competencias digitales sino, en especial, para consolidar los conocimientos sobre la enseńanza y el aprendizaje de la lectura y la escritura, sus perspectivas epistemológicas y contextos de aplicación; se precisan procesos de formación docente alternativos que permitan la construcción de nuevos saberes (Baez \& D’Ottavio, 2020; Guzmán, 2017; Silva-Peña et al., 2016).

Además, los aspectos socioemocionales resultaron un aspecto emergente en dos sentidos: primero, la pandemia significó pérdidas, duelo y desesperanza, lo que reveló oportuno atender las competencias socioemocionales en estudiantes y docentes; y, segundo, Mairena \& Vijil (2020) señalan la importancia de las interacciones sociales como el componente "más importante para la alfabetización exitosa de niños y niñas y particularmente de la comprensión, incluso más que los materiales o actividades usados durante las clases" (p. 52); en tanto, "los lectores y escritores se forman en comunidad, no en soledad" (Lerner, 2020, 17m43s). A pesar de esto, covid-19 impuso la distancia física entre estudiantes y docentes, por lo que el acompañamiento quedó a cargo de los integrantes de la familia, sin preparación previa y, en ocasiones, sin tiempo ni recursos para esto.

El presente trabajo muestra algunas limitantes resultado de la novedad del problema abordado y la consideración de un grupo de docentes con experiencia diversa, motivo por el cual se sugiere continuar el estudio ampliando el número de participantes, con trayectorias de mayor antigüedad y a partir de otras técnicas de recolección de datos que permitan profundizar sobre las prácticas de alfabetización. A fin de ampliar el conocimiento, se propone avanzar en la investigación sobre la alfabetización extraescolar realizadas en casa o en espacios comunitarios_-, y la alfabetización digital — desarrolladas en plataformas en línea, usualmente asociadas al juego-, el diseño de materiales y recursos digitales para la enseñanza de la lectura y la escritura, así como de las necesidades de formación para generar acciones de fortalecimiento docente frente a la alfabetización inicial y la educación a distancia.

\section{Referencias}

Aguilera, L. M. (27 de febrero de 2020). La escuela tiene que mirar el lenguaje de la vida: Lepe García. Noticias 48. La verdad cada mañana. https://bit.ly/32G8CCA

Alves, M. M. \& Barreto, S. (2006). Reforma educacional, intensificación del trabajo docente, cuidado y género. En M. Feldfeber, \& D. Andrade (eds.), Politicas educativas y trabajo docente. Nuevas regulaciones ¿nuevos sujetos? (pp. 181-204). NOVEDUC.

Baez, M. O. \& D’Ottavio, M. E. (2020). La diversidad en el aula: el desafío de interpretar la singularidad de los procesos de alfabetización inicial. Ciencia y Educación, 3(3), 31-40. https://doi.org/10.22206/cyed.2019.v3i3. pp31-40

Barton, D., \& Hamilton, M. (2000). Literacy Practices. En D. Barton, M. Hamilton, \& R. Ivanic, Situated Literacies. Reading and writing in context (pp. 7-15). Routledge. 
Brunetto, S. (26 de febrero de 2020). Coronavirus: aprender a leer y escribir en cuarentena. Página12. https://bit.ly/3qYQ0sb

Cervantes, E. \& Gutiérrez, P. R. (2020). Resistir la Covid-19. Intersecciones en la Educación de Ciudad Juárez, México. Revista Internacional de Educación para la Justicia Social, 9(3), 7-23. https://doi.org/10.15366/riejs2020.9.3.001

Chamberlain, L., Lacina, J., Bintz, W. P., Jimerson, J. B., Payne, K., \& Zingale, R. (2020). Literacy in Lockdown: Learning and Teaching During COVID-19 School Closures. The Reading Teacher, 74(3), 243-253. https://doi.org/ 10.1002/trtr. 1961

Comisión Nacional para la Mejora Continua de la Educación. (2020a). Experiencias de las comunidades educativas durante la contingencia sanitaria por covid-19. Educación básica. MEJOREDU. https://bit.ly/2ORmDu3

Comisión Nacional para la Mejora Continua de la Educación. (2020b). Docentes que leen y escriben. La lectura y la escritura en $1^{\circ}$ y $2^{\circ}$ grados de educación primaria. MEJOREDU. https:// bit.ly/2OWQ3XJ

Creswell, J. W. (2007). Qualitative inquiry y research design. Choosing among five approaches. SAGE Publications.

Creswell, J. W. (2014). Research Design. Qualitative, quantitative, and mixed methods approaches. SAGE Publications.

Espinosa, E. \& Mercado, R. (2008). Mediación social y apropiación de nuevas propuestas pedagógicas. Revista Latinoamericana de Estudios Educativos, XXXVIII(3-4), 201-232. https:// bit.ly/3xecFUW

Forteza-Forteza, D., Rodríguez-Martín, A., Álvarez-Arregui, E., \& Menéndez, D. (2021). Inclusion, dyslexia, emotional state and learning. Perceptions of Ibero-american children with dyslexia and their parents during the COVID-19 lockdown. Sustainability, 13(5), 2739. https:// doi.org/10.3390/su13052739
Galicia, A. (1978). El método global de análisis estructural para la enseñanza de la lectura y la escritura en el primer grado de educación primaria [Tesis de licenciatura, Universidad Pedagógica Nacional]. http://200.23.113.51/ pdf/123.pdf

Gobierno de México. (2020). Programa Sectorial de Educación 2020-2024. Diario Oficial de la Federación. https://bit.ly/3krvmye

González, O., González, M. \& Ruiz, J. C. (2012). Consideraciones éticas en la investigación pedagógica: una aproximación necesaria. EDUMECENTRO, 4(1), 1-5. https://bit.ly/3tIoQqA

Guzmán, R. J. (2017). Aprendizaje de los profesores sobre alfabetización y métodos de enseñanza. Folios, (46), 105-116. https://bit.ly/3kuh1kY

Hoz, G., Wallace, Y. \& Heredia, E. (2021). Alfabetizar a distancia en el inicio de la escuela primaria. Un análisis de propuestas publicadas por la provincia de Buenos Aires en Argentina. Revista Iberoamericana de Tecnología en Educación y Educación en Tecnología, (28), 282-293. https:// doi.org/10.24215/18509959.28.e35

Lerner, D. (26 de noviembre de 2020). La alfabetización en tiempos depandemia 2021-2021 [Archivo de Video]. YouTube. https://bit.ly/34A6YmX

Lerner, D. (2001). Leer y escribir en la escuela: lo real, lo posible y lo necesario. Fondo de Cultura Económica.

Lobe, B., Morgan, D., \& Hoffman, K. A. (2020). Qualitative data collection in an era of social distancing. International Journal of Qualitative Methods, 19, 1-8. https://doi.org/10.1177/ 1609406920937875

Mairena, D. \& Vijil, A. (2020). Lectoescritura inicial: una transición con sentido. Revista de la Universidad del Valle de Guatemala, 39(Especial), 51-61. https://bit.ly/3qZMxcU

Marcelo, C. (2008). El profesorado principiante. Inserción a la docencia. Ediciones Octaedro. https:// bit.ly/3ndwyGR

Martin, C. (08 de septiembre de 2020). Alfabetización en tiempos de Covid 19. Integratek. https:// bit.ly/3lrGfAS 
Mercado, R. (2018). Los saberes docentes como construcción social. La enseñanza centrada en los niños. Fondo de Cultura Económica.

Organización de las Naciones Unidas. (2020). Objetivo 4. Educación de calidad. Objetivos de Desarrollo Sostenible. https://bit.ly/3ksWVr3

Organización de las Naciones Unidas para la Educación, la Ciencia y la Cultura. (8 de septiembre de 2020). Día Internacional de la Alfabetización. https://bit.ly/2OPc9LJ

Organización de las Naciones Unidas para la Educación, la Ciencia y la Cultura. (2019). Marco de competencias de los docentes en materia de TIC. UNESCO. https://bit.ly/3rZ6YH3

Organización para la Cooperación y el Desarrollo Económico. (2019). Nota pais: Programa para la Evaluación Internacional de Alumnos (PISA). PISA 2018-Resultados. https://bit.ly/3bJXNUo

Sabariego, M., Massot, I. \& Dario, I. (2004). Métodos de investigación cualitativa. En R. Bisquerra, Metodología de la investigación educativa (pp. 293-328). La Muralla.

Sánchez, S. \& Santolària, A. (2020). Análisis de publicaciones sobre alfabetización inicial desde una perspectiva didáctica. Tejuelo: Didáctica de la Lengua y la Literatura, 32, 229-262. https://doi. org/10.17398/1988-8430.32.229

Secretaría de Bienestar. (2021). Decreto por el que se formula la Declaración de las Zonas de Atención Prioritaria para el año 2021. Diario Oficial de la Federación: https://bit.ly/3e0gx33
Secretaría de Educación Pública. (2017a). Modelo educativo para la educación obligatoria. Educar para la libertad y la creatividad. SEP. https:// bit.ly/3dtkL2D

Secretaría de Educación Pública. (2017b). Aprendizajes clave para la educación integral. Plan y programas de estudio para la educación básica. SEP. https:// bit.ly/2GXziYg

Secretaría de Educación y Deporte. (2020a, 17 de Julio). Diálogo FECHAC, y Organizaciones con el Secretario de Educación y Deporte [video]. YouTube. https://bit.ly/36FwzvE

Secretaría de Educación y Deporte. (2020b, $1^{\circ}$ de mayo). Avanza "Chihuahua, Educación a Distancia” [video]. YouTube. https://bit.ly/ 3f3wF3Q

Silva-Peña, I., Tapia, R. \& Ibáñez, M. (2016). Concepciones docentes sobre la escritura en primer año de educación básica. Paradígma, XXXVII(1), 46-60. https://bit.ly/3bHKmnJ

Yin, R. (2003). Case study research. Design and methods. SAGE Publications.

Zepeda, M. R. (2020). Análisis sobre la influencia del COVID-19 en la atención a alumnos con barreras para el aprendizaje y la participación social. En J. C. Yáñez, \& R. J. Alonso (eds.), Cuando enseñamos y aprendimos en casa. La pandemia en las escuelas de Colima (pp. 62-69). Puertabierta Editores. 